\title{
BMJ Open Pharmacists' roles in supporting people living with severe and persistent mental illness: a systematic review protocol
}

\author{
Sarira El-Den (D) , ${ }^{1}$ Sara S McMillan (D) ,, ${ }^{2,3}$ Amanda J Wheeler (i) , ${ }^{3,4}$ Ricki Ng (D) , ${ }^{1}$ \\ Helena Roennfeldt (D) , 3,5 Claire L O'Reilly (D) ${ }^{1}$
}

To cite: El-Den S, McMillan SS, Wheeler AJ, et al. Pharmacists' roles in supporting people living with severe and persistent mental illness: a systematic review protocol. BMJ Open 2020;10:e038270. doi:10.1136/ bmjopen-2020-038270

- Prepublication history and additional material for this paper are available online. To view these files, please visit the journal online (http://dx.doi. org/10.1136/bmjopen-2020038270).

Received 05 March 2020

Revised 12 May 2020

Accepted 15 May 2020

A Check for updates

C Author(s) (or their employer(s)) 2020. Re-use permitted under CC BY-NC. No commercial re-use. See rights and permissions. Published by BMJ.

${ }^{1}$ Sydney Pharmacy School, Faculty of Medicine and Health, University of Sydney, Sydney, New South Wales, Australia

${ }^{2}$ School of Pharmacy and Pharmacology, Griffith University, Southport, Queensland, Australia ${ }^{3}$ Menzies Health Institute, Griffith University, Nathan, Queensland, Australia

${ }^{4}$ Faculty of Health and Medical Sciences, University of Auckland, Auckland, New Zealand

${ }^{5}$ The University of Melbourne Centre for Psychiatric Nursing, Carlton, Victoria, Australia

Correspondence to

Dr Claire L O'Reilly;

claire.oreilly@sydney.edu.au

\section{ABSTRACT}

Introduction Severe and persistent mental illness (SPMI) can significantly impact a person's social, personal and professional life. Previous studies have demonstrated pharmacists' roles in mental healthcare; however, limited studies to date have focused on pharmacists' roles in providing healthcare services, specifically, to people living with SPMI. The aim of this systematic review is to explore the pharmacists' roles in providing support to people living with SPMI.

Methods and analysis A systematic search will be conducted in Medline, Embase (Ovid), PsycINFO, CINAHL, Web of Science, Scopus, Cochrane Library, International Pharmaceutical Abstracts and ProQuest Dissertations and Theses to identify potentially relevant primary research for inclusion. This will be guided by the Preferred Reporting Items for Systematic Review and Meta-Analysis Protocols checklist for systematic reviews. All primary research publications regardless of study design exploring or reporting on pharmacists' involvement in supporting people living with SPMI will be considered for inclusion. A tabular summary will be completed using data extracted from each included publication. Data synthesis and quality assessment methods will be chosen based on included study designs.

Ethics and dissemination The results will be published in a peer-reviewed journal and used to inform the development of a pharmacist-specific training package to support people living with SPMI.

PROSPERO registration number CRD42020170711.

\section{INTRODUCTION}

Severe and persistent mental illness (SPMI), including (but not limited to) schizophrenia and other psychotic disorders, bipolar disorder and severe and recurrent major depression, refers to any mental illness that has a continuous and significant lifelong impact on a person's relationships, social functioning, education and livelihood. ${ }^{1}$ People living with SPMI are at a considerable higher risk of premature death, in that they die 10-20 years earlier than the general population. ${ }^{23}$ For people living with schizophrenia, this gap in mortality has increased over time, mainly due to preventable physical

\section{Strengths and limitations of this study}

To our knowledge, this is the first systematic review investigating the roles of pharmacists in any healthcare setting, supporting people living with severe and persistent mental illness (SPMI).

- Inclusion of all study types will allow for a comprehensive overview of the literature relating to pharmacists' roles in supporting people living with SPMI.

- Broad inclusion criteria may present challenges for synthesising the evidence, but will allow for the identification of gaps in the existing literature.

- A limitation of this review is the potential to exclude relevant studies published in other languages, as this review will be restricted to articles published in English.

- Data extraction and quality assessment of included studies will be conducted by one member of the research team.

illnesses such as cardiovascular and metabolic disease, the side effects of second generation antipsychotic medicines used for treatment. $^{2}{ }^{4}$ Furthermore, people living with SPMI, such as schizophrenia, are also more likely to die by suicide. ${ }^{4}$ Moreover, smoking is up to three times more prevalent among people living with a mental illness, and up to five times more prevalent among people living with SPMI. ${ }^{5}$ Despite these known preventable risk factors, people living with SPMI receive poorer physical healthcare, compared with the general population. ${ }^{2}$ The WHO recognises that people living with SPMI require improved access to healthcare services to facilitate the recognition, diagnosis, treatment and monitoring of comorbid physical health problems. $^{2}$ Consequently, WHO guidelines highlight key priority areas for improvement including weight management, substance use disorders, cardiovascular disease and risk, diabetes, HIV/AIDS, infections and tobacco cessation. ${ }^{2}$ Hence, people living with SPMI require access to a range of healthcare professionals in order to manage 
their illness(es) and treatment(s), including primary healthcare professionals. ${ }^{2}$

Pharmacists are increasingly being recognised for their current and emerging roles in mental healthcare, as evidenced by guidelines, reports and frameworks which highlight their integral contribution to screening, initiating and maintaining treatment, improving medicine adherence and providing medicines information, in both primary and secondary care settings. ${ }^{6-8}$ This is reflected in the literature, whereby recent research has demonstrated that pharmacists are capable of screening to identify people at risk of depression and referring appropriately for diagnosis and therapy, ${ }^{9-11}$ managing psychotropic medication-related problems, ${ }^{12-15}$ providing antidepressant adherence support and working within multidisciplinary mental healthcare teams. ${ }^{16-18}$ However, research supporting pharmacists' roles in caring for people living with SPMI is lacking. Preliminary evidence indicates that pharmacists hold generally positive attitudes towards people living with SPMI; however, lack of time and confidence have been identified as barriers to their involvement in supporting this consumer population. ${ }^{19}$ Lack of confidence among healthcare professionals in supporting people experiencing mental illnesses and crises is not uncommon. For example, a recent systematic review exploring healthcare professionals' confidence in caring for people at risk of suicide identified that lack of confidence in this area was attributable to inadequate suicide education and training in healthcare curricula. ${ }^{20}$ Consequently, healthcare professionals often rely on upskilling through education and training programmes post-registration (eg, continuing professional development) to improve their knowledge of and confidence in supporting people experiencing mental health crises, such as, suicide. ${ }^{20}$

Given the accessibility of community pharmacies, pharmacists are in an ideal position to provide a triage-style role and refer/signpost consumers to relevant healthcare professionals and local services. Furthermore, given the physical illnesses that may arise as a result of the side effects of psychotropic medicines and symptoms of SPMI, pharmacists' roles as medicines experts enables them to support the physical healthcare of people living with SPMI. There is evidence to support the acceptability, feasibility and effectiveness of pharmacist-led interventions for a range of physical health conditions, including but not limited to tobacco cessation, ${ }^{21-23}$ alcohol use, ${ }^{24}$ cardiovascular risk, ${ }^{25} 26$ diabetes ${ }^{27} 28$ and infectious illnesses, ${ }^{29}$ among diverse populations. However, research exploring pharmacist-led risk assessment and service provision for people living with SPMI is lacking.

A 2018 Cochrane intervention review identified that non-dispensing pharmacist services can have a positive impact on consumers' health, with comparable outcomes to services delivered by other healthcare professionals. ${ }^{30}$ However, this Cochrane review did not solely focus on people living with SPMI. ${ }^{30}$ Other reviews have explored the literature pertaining to pharmacist-delivered interventions, for mental illnesses, generally, ${ }^{31}{ }^{32}$ and specifically for depression. ${ }^{334}$ There have also been literature reviews pertaining to pharmacists' roles in improving adherence to antidepressant medicines. ${ }^{35-37}$ However, there is no comprehensive literature review specifically exploring pharmacists' roles in providing services or care for people living with SPMI. Hence, the aim of this systematic review is to explore the literature surrounding the role of pharmacists in supporting people living with SPMI. Specifically, this systematic review will identify the types of services and interventions delivered by pharmacists for consumers living with SPMI and their impact on consumer outcomes.

\section{Research questions}

1. Have pharmacists been involved in providing support for people living with SPMI?

2. What is pharmacists' level of involvement; and nature, extent and outcomes of their support for people living with SPMI?

3. What type of education and training do pharmacists undertake prior to providing support for people living with SPMI?

4. What are the barriers to and facilitators of pharmacists' involvement in supporting people living with SPMI?

\section{METHODS AND ANALYSIS}

This systematic review will be guided by the Preferred Reporting Items for Systematic Review and Meta-Analysis Protocols (PRISMA-P) checklist. ${ }^{38}$ The PRISMA-P checklist was completed and can be seen in online supplementary appendix 1 .

\section{Database search}

Medline, Embase (Ovid), PsycINFO, CINAHL, Web of Science, Scopus, Cochrane Library, International Pharmaceutical Abstracts and ProQuest Dissertations and Theses will be searched systematically to identify publications for inclusion. The search will be based on two concepts relating to (1) pharmacy and (2) SPMI, using keywords and Medical Subject Headings (MeSH terms), where relevant, depending on the database.

The search strategy was developed by four members of the research team, in consultation with an academic librarian. The search strategy was informed by the aim of the systematic review, as well as, the WHO definition of severe mental disorders:

... a group of conditions that include moderate to severe depression, bipolar disorder, and schizophrenia and other psychotic disorders ${ }^{2}$

The search strategy was then discussed with other members of the research team, and pilot tested with another academic librarian prior to conducting the main searches. The final search was peer-reviewed by an academic librarian. A sample search strategy can be seen in online supplementary appendix 2 . The final search will 
be conducted by one research team member with experience in database searching.

\section{Screening}

Electronic search results will be downloaded into Endnote, duplicates will be removed automatically and manually, followed by screening of titles, abstracts and full-texts of potentially relevant citations. The first stage of screening involving duplicate removal and title screening will be conducted by one member of the research team. At the abstract stage, citations will be imported into the Covidence software. ${ }^{39}$ Two members of the research team will conduct the abstract and full-text screening process, within the Covidence systematic review software program. The results will be recorded in the program and disagreement at any stage of the screening process will be reconciled with a third member of the research team through discussion. The reference lists of included full-text citations will also be screened by these two members of the research team to identify any potentially relevant publications for inclusion. Any disagreements will be discussed and resolved through consultations with a third member of the research team.

\section{Eligibility criteria}

The title and abstract (or summary) of each exported citation will be included in the next stage of full-text screening if it meets the following criteria:

1. Published after 1990 (until 1 April 2020).

a. This date was chosen as it closely corresponds to the time when second generation antipsychotics were introduced $^{40}$ and there was a global shift towards community-based mental health care. ${ }^{41-43}$

2. Specifically refers to people living with SPMI, or mentions any of the following mental illnesses:

a. Schizophrenia.

b. Bipolar disorder.

c. Any psychotic illness (eg, schizoaffective disorder, psychotic depression).

d. Moderate-to-severe depression.

e. Moderate-to-severe anxiety.

f. Substance use disorders.

If a study reports on a mixed population of consumers which includes people living with SPMI, it will be considered for inclusion if the outcomes pertaining to pharmacist-led support for people living with SPMI, specifically, can be extracted.

3. Involves pharmacists or pharmacy staff or a multidisciplinary healthcare team which may involve pharmacists.

4. Refers to any setting where pharmacists may have a role in providing healthcare/services for consumers, such as:

a. Hospitals.

b. Community pharmacies.

c. Outpatient/community settings.

d. Aged care/residential facilities.
Citations will be included at this stage if they meet the above criteria, regardless of study design. All study designs will be considered for inclusion in this systematic review. Citations will be excluded at this stage if they describe any non-primary research, such as, letters, editorials, commentaries and protocols. Reviews of the literature will be excluded but first assessed for any potentially relevant individual studies that they have included.

Based on the above criteria, articles will be identified for full-text screening. This systematic review will include studies with any design and will not limit study inclusion to specific outcomes (ie, inclusion will not be limited to studies reporting on specific consumer-reported or pharmacist-reported outcomes, only). Hence, nonrandomised controlled trial study designs, including but not limited to implementation, observational and qualitative studies may be included. In addition to the criteria for title and abstract screening, articles will be considered for inclusion at the full-text screening stage if they meet the following criteria:

1. The study involves the delivery of a service by a pharmacist, pharmacy support staff member or pharmacy student, individually or as members of a broader healthcare team.

a. If a study reports on a mixed population of healthcare professionals, including pharmacists, pharmacy staff or pharmacy students, supporting people living with SPMI, the study will be included if the outcomes specifically pertaining to pharmacist-led support for people living with SPMI can be extracted.

2. The service is provided to consumers living with SPMI.

a. The study specifically states that the consumers were living with SPMI and/or

b. the consumer population is living with a psychotic illness (eg, psychotic depression, schizophrenia, schizoaffective disorder, bipolar disorder) and/or

c. the consumer population is living with moderateto-severe depression, anxiety or any other mental illness (with the exception of substance-use disorders, only) that is described as severe or persistent, in the manuscript. If those exact terms were not used, but the study provided a detailed description regarding the significant impact the mental illness caused to the person's life whereby it could be deduced that the illness would be categorised as an SPMI (ie, not mild depression that is chronic), then the study would be eligible for inclusion, provided that it met other inclusion criteria. Particular attention will be paid to studies that use the terms 'chronic', 'recurrent' and/or 'treatment-resistant' to describe the mental illness experienced by the study population.

3. The study occurs in any setting that involves providing healthcare/services for consumers living with SPMI.

a. For example, a study conducted in a university classroom setting would not be eligible for inclusion, but a study conducted in a teaching hospital would be 
eligible for inclusion.

4. The full-text of a peer-reviewed publication is available, regardless of study design type.

The following exclusion criteria will also be applied:

1. Publications reporting on non-primary research, such as editorials, letters, book chapters, case reports, protocols and commentaries.

2. Studies focusing solely on pharmacist services for people living with substance use disorders (eg, opioid, tobacco, alcohol) will be excluded. Pharmacists' roles in the provision of services (eg, naloxone dispensing, smoking cessation therapy, alcohol misuse screening) for people living with substance-use disorders have been established and explored previously through published reviews of the literature. ${ }^{2344-49}$

a. If study participants are living with an SPMI, as de-

fined in our inclusion criteria (above) and are also

experiencing a comorbid substance-use disorder, then outcomes pertaining to both the SPMI and the substance-use disorder will be reported.

3. Studies published in languages other than English.

\section{Data extraction}

A comprehensive overview of included studies will be presented, with detailed information provided about each included study relating to the SPMI, extent of pharmacist involvement, study setting and service/intervention provided. Data from each study will be reported in a tabular format including, but not limited to, information about the country where the study was undertaken, study aim, definition of SPMI used in the study, study design, study consumer and healthcare professional population(s), description of the intervention, nature and extent of pharmacist involvement in relation to the intervention and training received by pharmacists to deliver the intervention (if any). Furthermore, the following outcomes will be presented, when reported in included studies:

1. Consumer-reported outcomes.

2. Pharmacist-reported outcomes.

3. Outcomes attributable to pharmacist involvement.

a. For example: cost-effectiveness, qualitative evaluations and impact on other healthcare professionals.

Data extraction for reporting will be conducted by one member of the research team. Ambiguities relating to data extraction will be discussed with other members of the research team. Depending on the included study types, appropriate cross-checks will be conducted to ensure the accuracy of the extracted data.

\section{Data synthesis}

This systematic review aims to include all studies that meet the inclusion criteria. Hence, it is anticipated that publications employing a broad range of study designs will be included and it is not possible to determine which data synthesis method will be most appropriate, a priori. Therefore, the included studies will inform the selection of appropriate data synthesis methods. For example, if a significant proportion of studies are quantitative evaluations of interventions reporting on a specific outcome, a meta-analysis may be appropriate. However, if the majority of included studies identified are qualitative, a meta-synthesis may be deemed more appropriate. On the other hand, if included studies employ a variety of heterogeneous study designs, it may be more appropriate to employ a narrative synthesis. The Guidance on the Conduct of Narrative Synthesis in Systematic Reviews, developed by Popay et $a l^{\tilde{0} 0}$ provides guidance for the conduct of narrative reviews and may be used for data synthesis. A combination of different tools and techniques may be used to synthesise the data (where appropriate), including textual descriptions, tabulation, grouping and thematic/content analysis. The data synthesis approach will be discussed in consultation with the research team using an iterative and inductive approach. Furthermore, since a mixture of study types is expected a combination of data synthesis methods may be required.

\section{Consumer and public involvement}

This systematic review protocol was developed and coauthored by a team of health services researchers including pharmacists, individuals with lived experience of mental illness, as well as, individuals with experiences of caring for someone living with mental illness, thereby engaging the expertise of practitioners, consumers and carers. The definitions and terminology used in this manuscript have been reviewed by mental health consumers and modified according to their suggestions and preferences. People with lived experience and their carers will continue to be involved in future stages of this research, including the systematic review, and the development (eg, content development, resource identification) and delivery (eg, workshop material delivery) of training and education for community pharmacists, informed by the results of this systematic review. For example, they will be involved in the development, piloting and delivery of some components of the training package. Furthermore, they will be recruited as members of any committees or governance structures overseeing the broader research.

\section{Quality assessment}

This systematic review aims to provide a comprehensive overview of the literature pertaining to the nature and extent of pharmacists' involvement in providing healthcare/services for people living with SPMI. To meet this aim, we anticipate the inclusion of a broad range of study designs. Hence, it is not possible to determine which quality assessment tool is most appropriate until data extraction is complete. Quality assessment will be conducted using appropriate tools depending on study type. For example, the ROBINS- $I^{51}$ and RoB $2^{52}$ tools may be used for included studies assessing the impact of an intervention, as appropriate for non-randomised and randomised studies of interventions, respectively. The Mixed Method Appraisal Tool ${ }^{53}$ may be used if included studies employ mixed methods. 


\section{Patient and public involvement}

Patients and/or the public were involved in the design, or conduct, or reporting, or dissemination plans of this research. Refer to the Methods section for further details.

\section{ETHICS AND DISSEMINATION}

The findings of this systematic review will be disseminated through scholarly conference presentations and peer-reviewed publication(s). Furthermore, the findings of this systematic review will be used to inform the development of a pharmacist-specific education and training package to support people living with SPMI. This training and education package will be developed in consultation with people with a lived experience of mental illness, carers, multidisciplinary experts and representatives from professional pharmacy, medical and mental health organisations. Hence, the results of this systematic review, as well as, the input of clinical experts and consumers with lived experience will facilitate the development of the education and training package.

\section{CONCLUSION}

There is a need for a comprehensive review of pharmacists' roles in supporting people living with SPMI, to explore pharmacists' current roles in this area and the outcomes related to their involvement in service provision for people living with SPMI. This systematic review protocol describes the process that will be used to conduct the search for, as well as, screening, data extraction, data synthesis and quality assessment of studies exploring pharmacists' roles in supporting people living with SPMI. By including all studies that explore pharmacists' roles in SPMI, the resulting systematic review will provide a comprehensive overview of all the primary research conducted in this area, highlighting gaps in the current literature and building a solid foundation for training development and the establishment of pharmacist-led services and interventions for people living with SPMI.

Twitter Sarira El-Den @SariraEl_Den, Sara S McMillan @SaraSinclairMcM, Amanda JWheeler @AmandaJWheeler1, Ricki Ng @ng_ricki, Helena Roennfeldt @ HelenaRoennfel2 and Claire L 0'Reilly @oreilly_claire

Acknowledgements We acknowledge the contribution of both academic librarians who helped with the development and revision of the search strategy (Ms Bonnie Dixon from Griffith University and Dr Yulia Ulyannikova from The University of Sydney).

Contributors All authors have contributed significantly to this manuscript. The protocol was developed by SE-D, SSM, AJW and CLO. RN and HR reviewed and refined the research questions and search strategy. SE-D wrote the first draft of the manuscript. CLO, SSM, AJW, RN and HR contributed to writing and reviewing the manuscript. All authors have reviewed and approved the final version of the manuscript and will be involved in conducting the systematic review.

Funding The authors have not declared a specific grant for this research from any funding agency in the public, commercial or not-for-profit sectors.

Competing interests None declared.

Patient consent for publication Not required.

Provenance and peer review Not commissioned; externally peer reviewed.
Open access This is an open access article distributed in accordance with the Creative Commons Attribution Non Commercial (CC BY-NC 4.0) license, which permits others to distribute, remix, adapt, build upon this work non-commercially, and license their derivative works on different terms, provided the original work is properly cited, appropriate credit is given, any changes made indicated, and the use is non-commercial. See: http://creativecommons.org/licenses/by-nc/4.0/.

ORCID iDs

Sarira El-Den http://orcid.org/0000-0001-7500-3351

Sara S McMillan http://orcid.org/0000-0003-3427-4467

Amanda J Wheeler http://orcid.org/0000-0001-9755-674X

Ricki Ng http://orcid.org/0000-0002-1334-6487

Helena Roennfeldt http://orcid.org/0000-0002-4569-1226

Claire L O'Reilly http://orcid.org/0000-0001-6416-8150

\section{REFERENCES}

1 Carey MP, Carey KB. Behavioral research on the severe and persistent mental illnesses. Behav Ther 1999;30:345-53.

2 World Health Organisation. Guidelines for the management of physical health conditions in adults with severe mental disorders. Geneva: WHO, 2018.

3 Liu NH, Daumit GL, Dua T, et al. Excess mortality in persons with severe mental disorders: a multilevel intervention framework and priorities for clinical practice, policy and research agendas. World Psychiatry 2017;16:30-40.

4 Saha S, Chant D, McGrath J. A systematic review of mortality in schizophrenia: is the differential mortality gap worsening over time? Arch Gen Psychiatry 2007;64:1123-31.

5 Prochaska JJ, Das S, Young-Wolff KC. Smoking, mental illness, and public health. Annu Rev Public Health 2017;38:165-85.

6 International Pharmaceutical Federation (FIP). Focus on mental health: the contribution of the pharmacist. The Hague: International Pharmaceutical Federation, 2015.

7 Royal Pharmaceutical Society England. No health without mental health: how can pharmacy support people with mental health problems? London: Royal Pharmaceutical Society, 2018.

8 Pharmaceutical Society of Australia. Mental health care project. A framework for pharmacists as partners in mental health care. Deakin West, Australian Capital Territory: Pharmaceutical Society of Australia, 2013.

9 O'Reilly CL, Wong E, Chen TF. A feasibility study of community pharmacists performing depression screening services. Res Social Adm Pharm 2015;11:364-81.

10 Knox ED, Dopheide JA, Wincor MZ, et al. Depression screening in a university campus pharmacy: a pilot project. J Am Pharm Assoc 2006;46:502-6.

11 Rosser S, Frede S, Conrad WF, et al. Development, implementation, and evaluation of a pharmacist-conducted screening program for depression. J Am Pharm Assoc 2013;53:22-9.

12 Gisev N, Bell JS, O'Reilly CL, et al. An expert panel assessment of comprehensive medication reviews for clients of community mental health teams. Soc Psychiatry Psychiatr Epidemiol 2010;45:1071-9.

13 Nishtala PS, McLachlan AJ, Bell JS, et al. Psychotropic prescribing in long-term care facilities: impact of medication reviews and educational interventions. Am J Geriatr Psychiatry 2008;16:621-32.

14 Schmidt I, Claesson CB, Westerholm B, et al. The impact of regular multidisciplinary team interventions on psychotropic prescribing in Swedish nursing homes. J Am Geriatr Soc 1998;46:77-82.

15 McMillan SS, Kelly F, Hattingh HL, et al. The impact of a personcentred community pharmacy mental health medication support service on consumer outcomes. J Ment Health 2018;27:164-73.

16 Bell JS, Aslani P, McLachlan AJ, et al. Mental health case conferences in primary care: content and treatment decision making. Res Social Adm Pharm 2007;3:86-103.

17 Finley PR, Rens HR, Pont JT, et al. Impact of a collaborative pharmacy practice model on the treatment of depression in primary care. Am J Health Syst Pharm 2002;59:1518-26.

18 Gable KN, Stunson MJ. Clinical pharmacist interventions on an assertive community treatment team. Community Ment Health $\mathrm{J}$ 2010;46:351-5.

19 Watkins A, McKee J, Hughes C, et al. Community pharmacists' attitudes toward providing care and services to patients with severe and persistent mental illness. J Am Pharm Assoc 2017;57:S217-24.

20 Boukouvalas E, El-Den S, Murphy AL, et al. Exploring health care professionals' knowledge of, attitudes towards, and confidence in caring for people at risk of suicide: a systematic review. Arch Suicide Res 2019:1-31. 
21 Maguire TA, McElnay JC, Drummond A. A randomized controlled trial of a smoking cessation intervention based in community pharmacies. Addiction 2001;96:325-31.

22 Crealey GE, McElnay JC, Maguire TA, et al. Costs and effects associated with a community pharmacy-based smoking-cessation programme. Pharmacoeconomics 1998;14:323-33.

23 Dent LA, Harris KJ, Noonan CW. Tobacco interventions delivered by pharmacists: a summary and systematic review. Pharmacotherapy 2007;27:1040-51.

24 Brown S, Henderson E, Sullivan C. The feasibility and acceptability of the provision of alcohol screening and brief advice in pharmacies for women accessing emergency contraception: an evaluation study. BMC Public Health 2014;14:1139.

25 Peterson GM, Fitzmaurice KD, Kruup H, et al. Cardiovascular risk screening program in Australian community pharmacies. Pharm World Sci 2010;32:373-80.

26 Hourihan F, Krass I, Chen T. Rural community pharmacy: a feasible site for a health promotion and screening service for cardiovascular risk factors. Aust J Rural Health 2003;11:28-35.

27 Lindenmeyer A, Hearnshaw $\mathrm{H}$, Vermeire $\mathrm{E}$, et al. Interventions to improve adherence to medication in people with type 2 diabetes mellitus: a review of the literature on the role of pharmacists. J Clin Pharm Ther 2006;31:409-19.

28 Krass I, Taylor SJ, Smith C, et al. Impact on medication use and adherence of Australian pharmacists' diabetes care services. J Am Pharm Assoc 2005;45:33-40.

29 Gudka S, Marshall L, Creagh A, et al. To develop and measure the effectiveness and acceptability of a pharmacy-based Chlamydia screening intervention in Australia. BMJ Open 2013;3:e003338.

30 de Barra M, Scott CL, Scott NW, et al. Pharmacist services for non-hospitalised patients. Cochrane Database Syst Rev 2018;9:Cd013102.

31 Finley PR, Crismon ML, Rush AJ. Evaluating the impact of pharmacists in mental health: a systematic review. Pharmacotherapy 2003;23:1634-44.

32 Richardson TE, O'Reilly CL, Chen TF. A comprehensive review of the impact of clinical pharmacy services on patient outcomes in mental health. Int J Clin Pharm 2014;36:222-32.

33 Sampson SJ, Todd A, Walton N, et al. Pharmacy-based management for depression in adults. The Cochrane database of systematic reviews 2019;4.

34 Hattingh HL, Kelly F, Fowler J, et al. Implementation of a mental health medication management intervention in Australian community pharmacies: facilitators and challenges. Res Social Adm Pharm 2017;13:969-79.

35 Al-Jumah KA, Qureshi NA. Impact of pharmacist interventions on patients' adherence to antidepressants and patient-reported outcomes: a systematic review. Patient Prefer Adherence 2012;6:87-100.

36 Rubio-Valera M, Serrano-Blanco A, Magdalena-Belío J, et al. Effectiveness of pharmacist care in the improvement of adherence to antidepressants: a systematic review and meta-analysis. Ann Pharmacother 2011;45:39-48.

37 Chong WW, Aslani P, Chen TF. Effectiveness of interventions to improve antidepressant medication adherence: a systematic review. Int J Clin Pract 2011;65:954-75.

38 Moher D, Shamseer L, Clarke M, et al. Preferred reporting items for systematic review and meta-analysis protocols (PRISMA-P) 2015 statement. Syst Rev 2015;4:1.

39 Covidence systematic review software. Veritas health innovation. Melbourne, Australia. Available: www.covidence.org [Accessed April 30, 2020].

40 Tamminga CA. Treatment mechanisms: traditional and new antipsychotic drugs. Dialogues Clin Neurosci 2000;2:281-6.

41 Robson B. From mental hygiene to community mental health: psychiatrists and Victorian public administration from the 1940s to 1990s. Provenance: The Journal of Public Record Office Victoria2008;7.

42 Mechanic D. Mental health services then and now. Health Aff 2007;26:1548-50.

43 Not For Service. Experiences of injustice and despair in mental health care in Australia. Canberra: Mental Health Council of Australia, 2005.

44 Mdege ND, Chindove S. Effectiveness of tobacco use cessation interventions delivered by pharmacy personnel: a systematic review. Res Social Adm Pharm 2014;10:21-44.

45 Greenhalgh T, Macfarlane F, Steed L, et al. What works for whom in pharmacist-led smoking cessation support: realist review. BMC Med 2016;14:209.

46 Saba M, Diep J, Saini B, et al. Meta-Analysis of the effectiveness of smoking cessation interventions in community pharmacy. J Clin Pharm Ther 2014;39:240-7.

47 Watson MC, Blenkinsopp A. The feasibility of providing community pharmacy-based services for alcohol misuse: a literature review. Int $J$ Pharm Pract 2009;17:199-205.

48 Nielsen S, Van Hout MC. What is known about community pharmacy supply of naloxone? A scoping review. Int J Drug Policy 2016;32:24-33.

49 Thakur T, Frey M, Chewning B. Pharmacist roles, training, and perceived barriers in naloxone dispensing: a systematic review. J Am Pharm Assoc 2020;60:178-94.

50 Popay J, Roberts H, Sowden A, et al. Guidance on the conduct of narrative synthesis in systematic reviews: a product from the ESRC methods programme. Lancaster: Lancaster University, 2006.

51 Sterne JA, Hernán MA, Reeves BC, et al. ROBINS-I: a tool for assessing risk of bias in non-randomised studies of interventions. BMJ 2016;355:i4919.

52 Higgins JPT, Sterne JAC, Savovic J, et al. A revised tool for assessing risk of bias in randomized trials. Cochrane Database Syst Rev 2016;10:29-31.

53 Hong QN, Fàbregues S, Bartlett G, et al. The mixed methods appraisal tool (MMAT) version 2018 for information professionals and researchers. Education for Information 2018;34:285-91. 